\title{
Orion Crew Member Injury Predictions during Land and Water Landings
}

\author{
Charles Lawrence ${ }^{1}$, Justin D. Littell ${ }^{2}$, Edwin L. Fasanella ${ }^{3}$, Ala Tabiei ${ }^{4}$ \\ ${ }^{1}$ NASA Glenn Research Center, Cleveland, Ohio: Lawrence@nasa.gov \\ ${ }^{2}$ University of Akron, Akron, Ohio: Justin.D.Littell@grc.nasa.gov \\ ${ }^{3}$ NASA Langley Research Center, Hampton, Virginia: Edwin.L.Fasanella@nasa.gov \\ ${ }^{4}$ Multi-Physics Simulation Engineering, Mason, Ohio: ATabiei@aol.com
}

\begin{abstract}
A review of astronaut whole body impact tolerance is discussed for land or water landings of the next generation manned space capsule named Orion. LS-DYNA simulations of Orion capsule landings are performed to produce a low, moderate, and high probability of injury. The paper evaluates finite element (FE) seat and occupant simulations for assessing injury risk for the Orion crew and compares these simulations to whole body injury models commonly referred to as the Brinkley criteria. The FE seat and crash dummy models allow for varying the occupant restraint systems, cushion materials, side constraints, flailing of limbs, and detailed seat/occupant interactions to minimize landing injuries to the crew. The FE crash test dummies used in conjunction with the Brinkley criteria provides a useful set of tools for predicting potential crew injuries during vehicle landings.
\end{abstract}

\section{Introduction and Background}

The NASA Constellation Program plans are to replace the space shuttle orbiter with a Crew Exploration Vehicle (CEV) Command Module (CM) named "Orion". Orion will be similar to Apollo but is larger and will carry a crew of four to six members while Apollo was designed for three astronauts. The Orion module will descend to Earth with a three-parachute recovery system in the event of emergency escape during the launch phase of the mission or during the final phase of the module's return to Earth. The module is to be designed to be capable of a primary water landing as well as land landings. Landing and recovery and protection of the crew members during water or land landings is a design challenge and despite years of successful space missions cannot be considered routine.

To keep the size of parachute systems reasonable and to optimize the weight of the landing system, the terminal velocity of the Orion module parachute recovery systems is to be designed to provide a descent velocity at landing of approximately $24 \mathrm{ft} / \mathrm{s}$ nominal to $33 \mathrm{ft} / \mathrm{s}$ off-nominal $(16-22.5 \mathrm{mi} / \mathrm{hr})$. In addition to the terminal descent (vertical) velocity, the parachute and module will move horizontally depending on the horizontal wind velocity. The velocities of the module at touch down are typical of crash impact velocities of small aircraft and helicopters. Thus, these impact velocities may produce injuries without some type of mitigation for impacts onto land. Under 


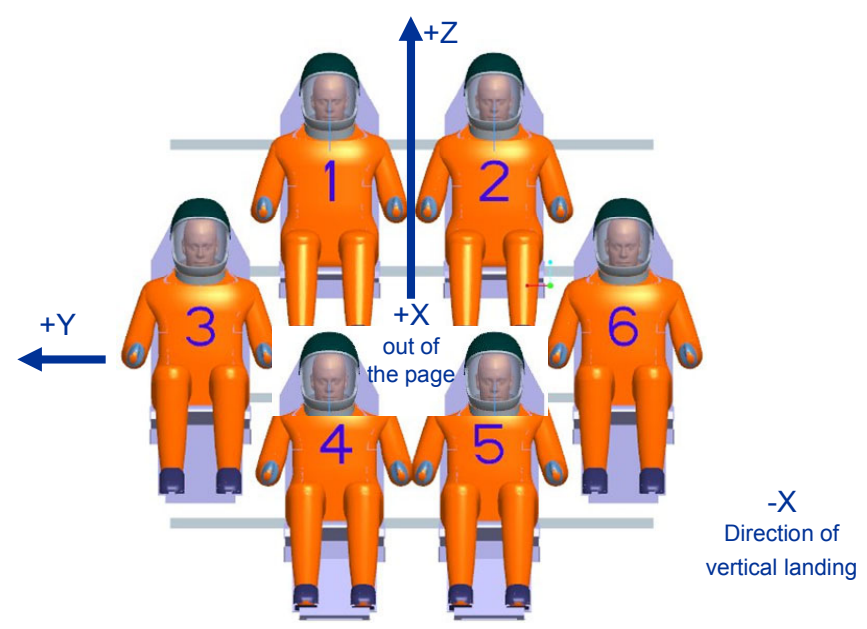

Figure 1. Six Crew Configuration and local $X, Y$, and Z-axes.

ideal nominal conditions, either a land landing with retro-rockets or impact attenuating airbags or a water landing will result in tolerable accelerations to the crew if the seats and restraint systems are properly designed. For off-nominal landing conditions, a stroking crew seat pallet similar to the Apollo design is planned for the Orion module to reduce the impact accelerations to conditions that are tolerable to the astronauts and that will prevent injury.

The primary purpose of this paper is to review current whole body human impact tolerance models, including the Brinkley model, used by the Constellation Program for assessing injury risk during nominal and off-nominal landings of Orion. In addition, the paper will discuss the use of finite element (FE) seat and occupant simulations for assessing injury risk for Orion. With properly designed seats with side supports and restraints, even relatively high accelerations can be tolerated with no or minimal injury, especially for impacts with the astronauts lying on their backs in a seat oriented as shown in Figure 1.

\section{Mathematical Lumped Parameter Injury Models}

The most effective human model for ejection seat design is the Dynamic Response Index (DRI) described by Stech and Payne in 1966. This work was performed for what is now called the Air Force Research Laboratory (AFRL) and was based on pioneering work in human biodynamics by von Gierke (1967) and his colleagues. The model was developed using data collected from vibration tests using volunteer subjects. The stiffness and breaking strength of the model were initially determined from tests of the vertebrae of cadavers. The overall frequency response was determined on the basis of the observed frequency response of the volunteers in laboratory experiments. The DRI model estimates of spinal injury rates were compared to actual operational ejection seat occupant spinal injury data by Brinkley (1968) and Brinkley and Shaffer (1971). On the basis of its validation using operation data, the Aeronautical Systems Center accredited the DRI model and incorporated it into the U.S. Air Force specification used to develop escape systems and to qualify ejection catapults. In the mid 1970s, the model was accredited by the Air Standardization Coordination Committee and incorporated into an Air Standard ratified by air services of Australia, Canada, New Zealand, the United Kingdom, and 
the Air Force and Navy of the United States. The model was integrated into a computer program with limits for the other orthogonal axes, which were based upon then current estimates of acceleration and rate of onset limits. This program was used throughout the 1970 s to evaluate the performance and to qualify U.S. Air Force escape systems.

\section{The Multi-Axial Dynamic Response Criteria}

The impact exposure criteria that are included in the Brinkley model are similar in the technical approach to the models that have been developed to specify the human limits for human exposure to vibration and noise. These models are intended to specify the limits of the motion of vehicles at or near the position of the human occupant to prevent injury or performance decrements. The models and their associated limits are based upon whole-body responses or body organ responses to mechanical forces. The Brinkley model is a method that provides limits for vehicle accelerations occurring in any vector direction. The models for each axis represent the primary resonances that have been judged to be associated with the tolerance end points that have been reported in experiments with volunteers or observed in operational incidents. The criteria that have been provided to NASA are based upon the presumption that the crew seats and restraint systems will be similar to the Apollo crew seats and restraint systems. Higher dynamic response limits may be appropriate if a more robust crew protection system such as used in the Soyuz crew module or such as those that were used to explore the response to multi-directional impact for Project Apollo cited earlier. A brief description of the Multi-axial Dynamic Response Method Criteria (referred to at NASA as the Brinkley Dynamic Response Model or Brinkley criteria) follows.

The equations of motion of the lumped parameter models that are used in the method are three second-order differential equations. Each equation is simply a forced springmass harmonic oscillator with damping. The forcing function " $A$ " in Equation (1) is the measured acceleration along each of the three seat axes. As a simplifying condition, the three equations are considered to be uncoupled.

$$
\ddot{x}+2 \xi \omega_{n} \dot{x}+\omega_{n}^{2} x=A
$$

Where:

$\ddot{x}$ is the relative acceleration of the dynamic system in each of the $\mathrm{X}, \mathrm{Y}$, or $\mathrm{Z}$ axis.

$\dot{x}$ is the relative velocity.

$x$ is the relative deflection where a positive value represents compression.

$\xi$ is the damping coefficient ratio.

$\omega_{n}$ is the undamped natural frequency of the dynamic system.

$A$ is the component of the measured acceleration along the specified axis. Since the seat axis is not an inertial frame, rotational acceleration must be considered in terms of the linear components of the angular motion. 
The dynamic response for each axis is given by:

$$
D R=\omega_{n}^{2} x / g
$$

Where $D R(\mathrm{t})$ is the response of the dynamic system and $g$ is the acceleration of gravity. Note that $D R$ is dimensionless since it is divided by $g$, the acceleration of gravity. The value " $x$ " when used along the $+Z$ axis is the compression of the spine. Note that $D R(\mathrm{t})$ is essentially the normalized response of the occupant to the input acceleration in $g$. The maximum value of $D R(\mathrm{t})$ for any axis should be less than the limiting value. Thus, $D R(\mathrm{t})$ can be plotted versus the input acceleration time history. The maximum of the response $D R(\mathrm{t})$ can be greater or less than the maximum of the input acceleration $A(\mathrm{t})$ depending on whether the simple harmonic oscillator amplifies or attenuates the driving acceleration. The following values for $\omega_{n}$ and $\xi$ shall be used:

$$
\begin{gathered}
\omega_{n x}=62.8, \omega_{n y}=58.0, \omega_{n z}=52.9 \\
\xi_{x}=0.2, \xi_{y}=0.09, \xi_{z}=0.224
\end{gathered}
$$

Limiting values of Dynamic Response, $D R$, levels have been set by NASA for Orion and they are provided in NASA CxP 70024.

The Brinkley model is limited in that it uses a simplified mechanical model of the dynamic whole-body response of the human. A schematic of the physical layout of the Brinkley model, showing the driving point for the model located in the midthorax, is shown in Figure 2. The nature of the Brinkley model and its representation of the human body as a lumped parameter model acting at a single point preclude the model from distinguishing relative movement of individual body parts. While this model works reasonably well for vertical z-axis loads, such as those of an ejection seat, where the major injury location is in the lower spine and the human body moves as a unit, it does not properly physically model some other impacts, such as side impacts, where sections of the body like the head relative to the body center of mass. In addition to the Brinkley model not having the capability to discern different types of injuries, the Brinkley model cannot provide insight into the effect of any advanced restraint system that provides additional restrictions or protection to the head or other parts of the body.
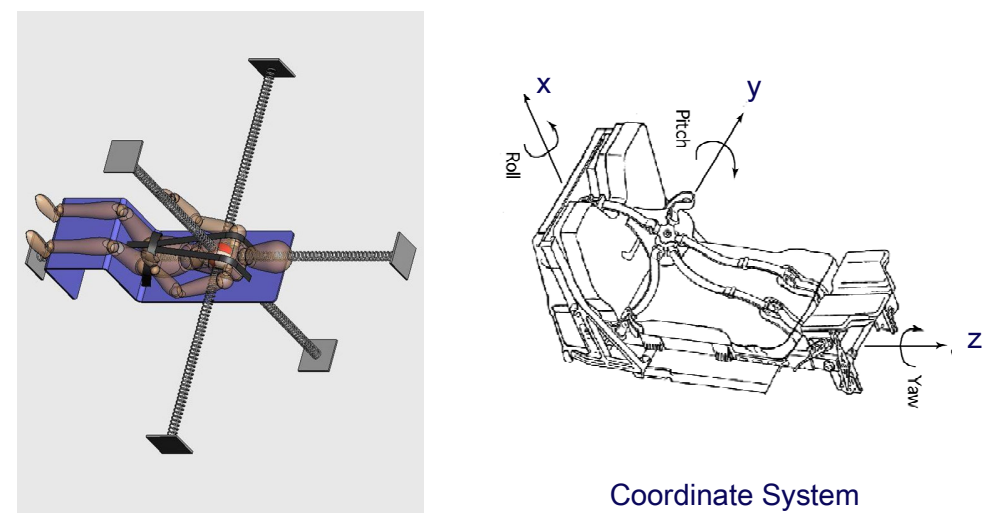

Coordinate System

Figure 2. Brinkley Single Degree of Freedom Body Model. 


\section{Other Impact Acceleration Criteria}

In contrast to the Air Force, the Army developed design guides requiring features such as crashworthy cockpits and seats to protect the crew in the event of a "survivable crash" (Coltman et al., 1989). The automobile industry was faced with federal regulations to design and build safer cars. Anthropomorphic Test Dummies (ATD's) with more human impact fidelity such as the Hybrid III were developed as instrumented passengers for controlled crash tests. The National Highway Traffic Safety Administration (NHTSA) regulates automobile safety and also sponsors research to make automobiles safer. A description of many of the injury criteria used in the automotive industry is provided in Schmitt, K.-U., et al.

The current state of the art does not allow the direct modeling and simulation of a human body subjected to injurious high acceleration loading or impact conditions. To address this limitation the automotive industry has attempted to generate a relationship between the physical behavior of the various crash test dummies and the human body. This has been attempted via a combination of cadaver, sub-injury level testing on human volunteers, animal experimentation and data gathering from actual accidents. While the automotive industry has years of experience correlating test crash dummy behavior during controlled automobile crashes with actual real world crashes and human injury little work has been done to correlate the dummy behavior with the impacts and accelerations expected to occur during Orion landings.

In the present effort the Hybrid III FEA model was selected as the baseline. This is a model of the most commonly used crash test dummies and was thought to be a good starting point for comparing the results from the crash test dummies to those from the Brinkley model. It was also thought that the Hybrid III was a reasonable crash test dummy for assessing alternate crew protection concepts. It is recognized that for situations such as side and rear impact models such as the Side Impact Dummy (SID) or Rear Impact Dummy (RID) are more suitable, however, the incorporation of these models is left for future work.

\section{Finite Element Crash Test Dummy Simulations}

The Orion (version 604) Crew Module (Fig. 3) was used to generate vehicle responses so that a comparison could be made between injury predictions from the Brinkley model and predictions from the FE crash test dummy. To generate the response, the vehicle was oriented in three primary human body directions; eyes in/out ( $\mathrm{z}$ axis), sideways (y axis) and spinal (z direction). The vehicle was given an initial velocity just before impact with the ground. These orientation exaggerate realistic landing scenarios, however, they provide extreme cases for comparing the Brinkley model to the crash test dummy results. The finite element program, LSDYNA was used to perform the analysis of the vehicle impacting ground and resulting acceleration profiles were computed and extracted at the location of the center of mass of the crew members. The extracted acceleration profiles were then input into the Brinkley model and the level of injury was computed using the Brinkley injury criteria. The initial impact velocity was adjusted until the velocities corresponding to high, medium and low injury risk were identified. Once the impact velocities corresponding to the three levels of injury risk associated with the Brinkley model were determined, and the acceleration profiles generated (for example Figure 4) a separate LS-DYNA model was created that included only the FE crash 


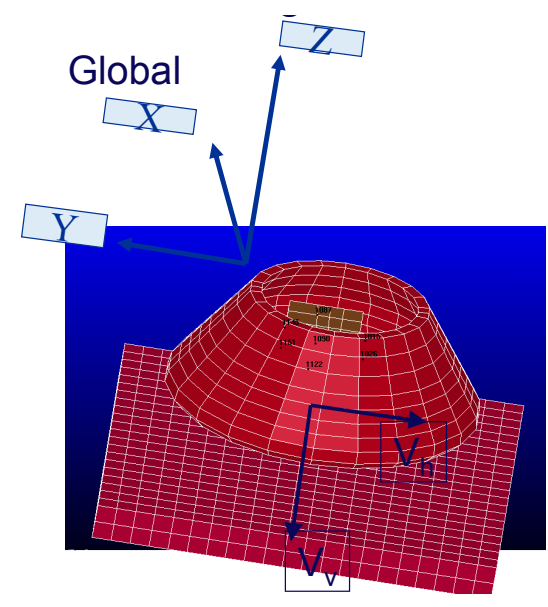

a) Seat Attenuation Model (Global Coordinate System)



b) Seat Attenuation Model (Brinkley Local Coordinate System) (Pressure Vessel not Shown)

Figure 3. 604 Seat Attenuation Model.

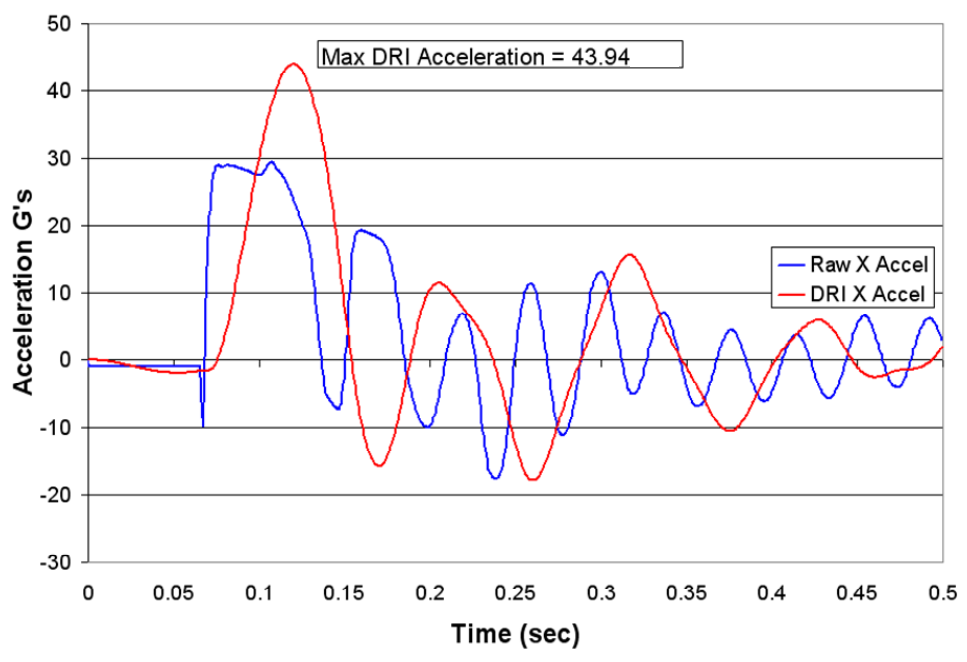

Figure 4. Acceleration Profile for High Eyes In/Out DR $(\mathrm{Vn}=\mathbf{5 8 . 3 3} \mathrm{fps})$.

test dummy constrained in a seat with a five point harness. The acceleration profiles were then applied to the seat in this model, simulations were run, and a comparison was made between the Brinkley injury criteria and the response extracted from the FE crash test dummy.

The FE crash test dummy provides a far greater amount of information concerning human body response including actual motions of the body such as limb flailing and head motion as well as loads within the body such as acceleration levels at specific locations in the body and forces on individual body parts (for example Figure 5). Industry standards have been established that minimize injuries by limiting allowable predicted accelerations, forces and moments in the crash test dummy. As previously mentioned in this report, it is important to note that many of the industry accepted standards have been developed for the automotive industry, or for other applications such as ejection seats, and may not be directly applicable to the type of conditions and 


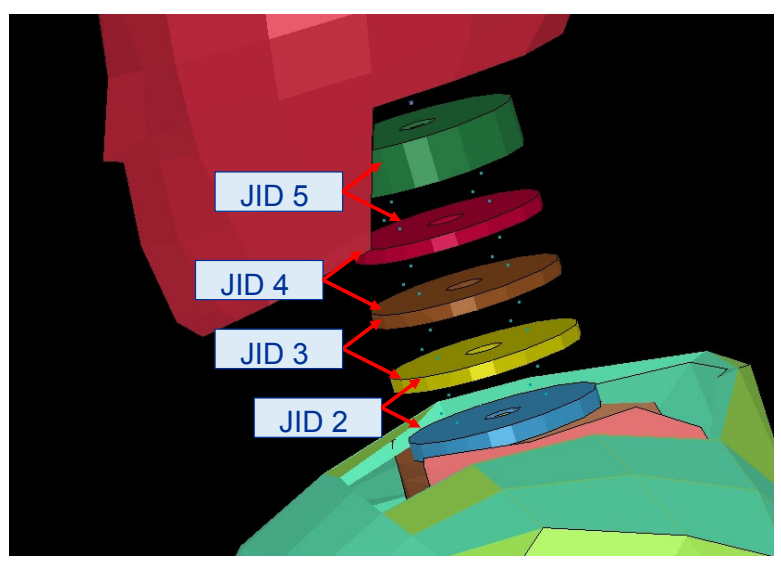

Figure 5. Location of Neck Joints.

injuries expected for the Orion crew members without further study and modification. It is also important to note that the FE test dummy is an analytical model of the physical dummy which is made up of non-human parts made from materials such as steel, aluminum, rubber and plastics. The FE test dummy is designed to predict the response of the physical test dummy and does not directly predict how a human might respond during an actual impact or the forces generated within the human body.

\section{Z-Axis (Spinal) Acceleration Simulations and Results}

Loading in the z-axis (spinal) direction was examined since this is the loading direction that the Brinkley model was originally designed to address and where the Brinkley model is most accurate. Since the results of the Brinkley model are well anchored in actual data for loading in the spinal direction, it was useful to compare the Brinkley predictions to the results generated from the FE crash test dummy and to industry accepted injury criteria. Figure 6 and Table 1 provide representative output from the LS-Dyna simulations. Figure 6 shows two intermediate time steps from the simulation. The first frame shows the maximum head flexion, which occurs just after the impact with the ground and the second frame shows maximum head extension, which occurs during the time period where the vehicle rebounds off of the ground. Table 1 provides a comparison among the three Brinkley injury levels and a collection of injury criteria (Schmitt, K.-U., 2004). Allowable limits for the injury criteria are included in the Table and conditions where the limits are exceeded are identified by red. The Brinkley injury risk criteria are consistent for low levels of injury in that none of the injury criteria allowable are exceeded. For medium Brinkley injury risk the allowable thorax acceleration limit is exceeded while the remainder of the injury criteria is within allowable limits. For high injury risk for the Brinkley model, the thorax acceleration is close to double the allowable limit and the neck moment extension allowable limit is exceeded. It is not surprising that the allowable neck moment is exceeded considering the head is not constrained and undergoes considerable motion during impact. It is interesting that the lumbar force is within allowable limits for all three Brinkley injury levels considering that for this direction of loading spinal cord injuries are expected to be most prevalent (Bowman, 1993). It is important to note that many of the industry accepted standards have been developed for the automotive industry, or for other applications such as ejection seats, and may not be directly applicable to the type of conditions and injuries expected for the Orion 

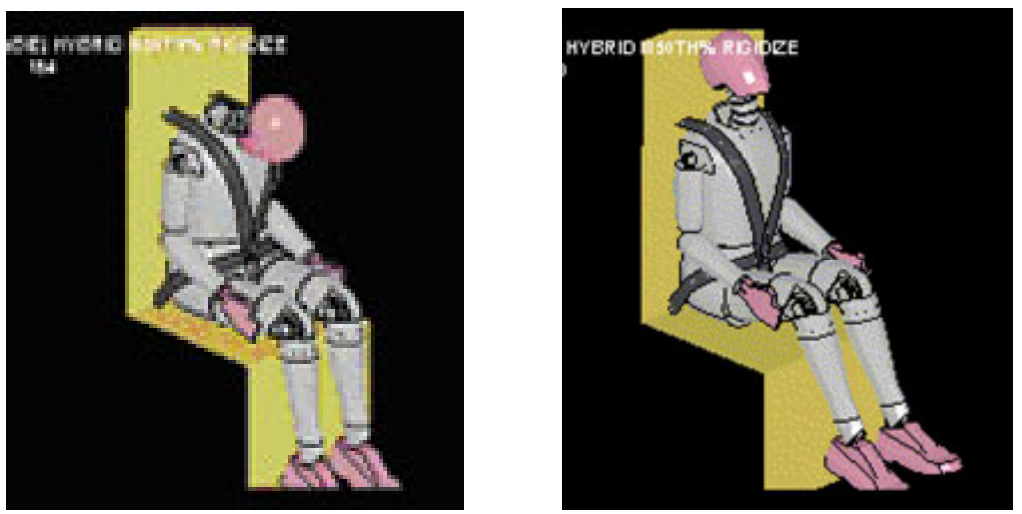

Figure 6. Hybrid III Crash Test Dummy Response at Intermediate Time Steps.

\begin{tabular}{|c|c|c|c|c|c|}
\hline Criteria $^{1}$ & Location & Allowable & $\begin{array}{l}\text { Brinkley Low } \\
\text { Injury }\end{array}$ & $\begin{array}{l}\text { Brinkley Medium } \\
\text { Injury }\end{array}$ & $\begin{array}{l}\text { Brinkley High } \\
\text { Injury }\end{array}$ \\
\hline $\begin{array}{l}\text { Head Injury Criteria } \\
\text { (HIC) }\end{array}$ & $\mathrm{N \#} 1 \mathrm{HIC}$ & 700 & 3.4 & 4.57 & 59.54 \\
\hline $\begin{array}{l}\text { Chest Severity Index } \\
\text { (CSI) }\end{array}$ & N\# 1787 CSI & 700 & 31.6 & 43 & 64.49 \\
\hline Thorax G's & N\# 1787 -resultant & $60 \mathrm{G}$ 's & $46.17 \mathrm{G}$ 's & $75.27 \mathrm{G}$ 's & $127.7 \mathrm{G}$ 's \\
\hline Pelvis G's & N\# 7501 - resultant & & $32.7 \mathrm{G}$ 's & 49.42 & $41.9 \mathrm{G}$ 's \\
\hline \multirow[t]{2}{*}{ Lumbar Force } & JT\# 26-37 -resultant & $6672 \mathrm{~N}$ & $2804 \mathrm{~N}$ & $4037 \mathrm{~N}$ & $4545 \mathrm{~N}$ \\
\hline & JT\# 38 -resultant & $6672 \mathrm{~N}$ & $4080 \mathrm{~N}$ & $4500 \mathrm{~N}$ & $5500 \mathrm{~N}$ \\
\hline Lumbar Moment & JSR\# 15 - & & $131.4 \mathrm{NM}$ & $143 \mathrm{NM}$ & 124.3 NM \\
\hline Neck Force & JT\# 2-5 - resultant & $6806 \mathrm{~N}$ & $887 \mathrm{~N}$ & $698 \mathrm{~N}$ & $1277 \mathrm{~N}$ \\
\hline $\begin{array}{l}\text { Neck Moment Flexion } \\
(-)\end{array}$ & JSR\# 16 - & $310 \mathrm{NM}$ & $66.42 \mathrm{NM}$ & $78.6 \mathrm{NM}$ & $79.42 \mathrm{NM}$ \\
\hline $\begin{array}{l}\text { Neck Moment } \\
\text { Extension }(+)\end{array}$ & JSR\# 17 - & $135 \mathrm{NM}$ & $108.6 \mathrm{NM}$ & $130 \mathrm{NM}$ & $184 \mathrm{NM}$ \\
\hline
\end{tabular}

${ }^{1}$ Trauma Biomechanics: Introduction to Accidental Injury, K.U. Schmitt, P. Niederer, F. Walz, Springer 2004

Table 1. Hybrid III Response and Brinkley Injury Levels Z Axis (Spinal) Excitation.

crew members. For example, many of the automotive injury criteria were developed for frontal impacts where the human body rapidly decelerates and body parts such as the head impacts some part of the automobile interior. Similarly, aircraft ejection seat criteria are designed for an upright seated pilot being ejected vertically through the aircraft canopy where the primary loading is up through the spine. For Orion nominal landings, the crew members will be seated on their backs and the primary loading will be in a direction from the crew member's back towards the chest. Further study is recommended before existing industry accepted injury criteria developed for applications such as automotive or military are used to assess Orion crew member injury.

\section{Y-Axis (Sideways) Acceleration Simulations and Results}

Results of FEA simulations for the standard five-point harness were compared with simulations that contained a variety of lateral supports inspired by the example of a modern racecar seat (Fig. 7). Modern racecar seats provide excellent driver protection during impacts that might normally be fatal with a more traditional seat design (Gramling, H., et al., Melvin, J.W, et al.). These seats provide a higher level of protection through the use of lateral supports, and head and neck restraint systems, as well as improved harnesses. Much of the improvements that have been realized in 


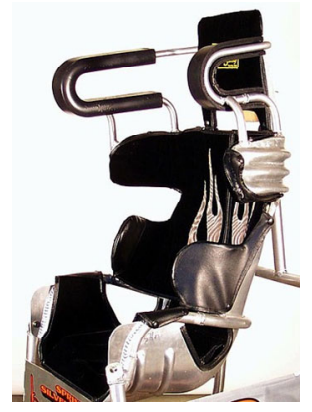

Modern racecar seat

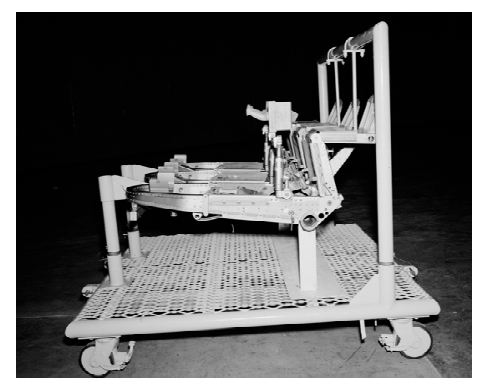

Apollo seat

(crew member seated on back)

Figure 7. Comparison between modern racecar and Apollo seat.

racecar seats are the results of trial and error and results obtained during actual operations and accidents. Orion seat designs cannot be designed via trial and error or in-use test programs and therefore to take advantage of any improvements in restraint or seat designs physical and FE crash test dummies will need to be used.

Simulations for impacts in the direction of the $y$-axis were conducted using the same Hybrid III FE model, harness and seat as was used for the Z-axis study in the previous section. The purpose of performing simulations in the sideways direction was to evaluate the effect of various combinations of side impact constraint devices on crewmember injury and to assess the general effectiveness of the FE crash test dummy for performing this type of study. The Hybrid III FE crash test dummy is not ideally suited for simulating side impacts; however, since this model was already generated for the work performed in the previous section it was expedient to re-use the model for side impacts. The results presented in this section may not be as accurate as if they had been computed with a better-suited dummy such as the Side Impact Dummy (SID) however, for performing a first order comparison of the effect of side constraints this model was adequate.

Figure 8 shows the four of the five configurations that were used to assess the effectiveness of side impact constraints. The baseline configuration was an unconstrained crewmember in the five-point harness. The second configuration was comprised of thin pads with a three-inch gap between the crewmember's head and shoulders. The third configuration (not shown in figure) was thicker pads with a oneinch gap between the crewmember's head and shoulder. The fourth configuration was comprised of thick pads in direct contact with the crew members head and shoulders and the fifth configuration was identical to the fourth with thick pads and hand and feet constraints were added to retain the crew member's arms and legs from significant flaying. The properties and thickness of the various pads are important for detailed analysis however for this preliminary study the pads were not optimized and the results are only indicative of overall trends. All of the simulations performed in this section of the report were done using the input acceleration profile that generated a high injury risk using the Brinkley model in the sideways direction. 


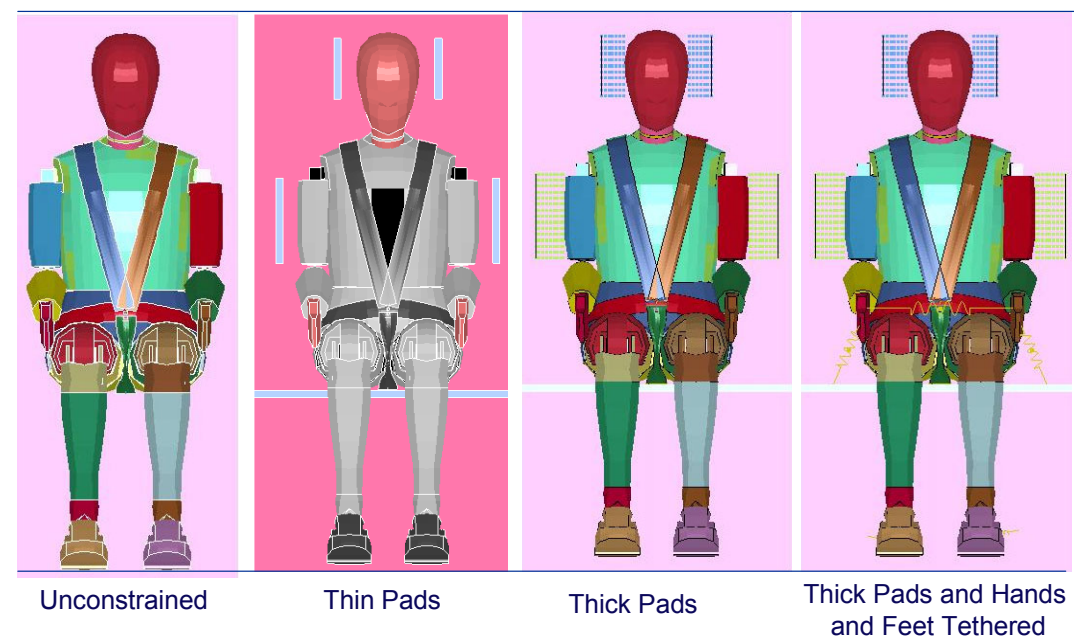

Figure 8. Comparison Among Padded and Constrained Crew Member for Sideways Loading.

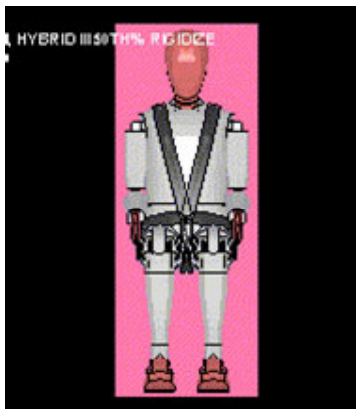

Initial Conditions

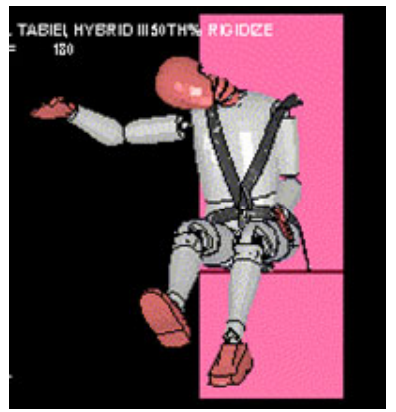

Initial Impact

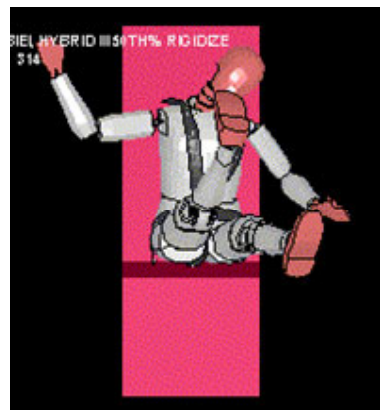

Rebound

Figure 9. Hybrid III Crash Test Dummy Response at Intermediate Time Steps for Side Impact (No Side Padding).

Figure 9 and Table 2 show the results for the first configuration where the crewmember is only constrained in the five-point harness. Figure 9 shows the crash test dummy response at three intermediate time steps for the model without any side padding. Large excursions of the head, arms and legs are shown clearly during both the initial impact and the rebound. The results in Table 2 agree with the Brinkley prediction of high injury risk considering that four of the seven injury criteria are exceeded including the Chest Severity Index (CSI), the thorax maximum allowable G and the neck flexion and extension. It is important to point out that the neck injury criteria are intended for frontal impacts and have been very loosely applied to the present application of side impact. For side impact there are no clearly defined neck moment criteria however it was felt that the frontal impact neck criteria could be reasonably applied, as an approximation, to side impacts. Additional study of neck injury criteria is certainly required before firm criteria can be established. 


\begin{tabular}{|c|c|c|c|c|c|c|}
\hline & Allowable & Five Point Harness* & Thin pads & $\begin{array}{l}\text { Thicker pads } \\
\text { 1" gap }\end{array}$ & Thick pads & $\begin{array}{l}\text { Thick Pads } \\
\text { and hand } \\
\text { and Feet } \\
\text { Constraints }\end{array}$ \\
\hline $\begin{array}{c}\text { HIC } \\
\text { (Node 1) }\end{array}$ & 700 & 225 & 4050 & 256 & 174 & 92 \\
\hline $\begin{array}{c}\text { CSI } \\
\text { (Node 1787) } \\
\end{array}$ & 700 & 965 & 1960 & 1965 & 1211 & 1559 \\
\hline $\begin{array}{c}\text { Thorax g's } \\
\text { (Node } 1787 \text { filtered @180 Hz) }\end{array}$ & $60 \mathrm{~g}$ 's & 78 g's & 90 g's & $62 \mathrm{~g}$ 's & $71 \mathrm{~g}$ 's & $81 \mathrm{~g}$ 's \\
\hline $\begin{array}{c}\text { Pelvis g's } \\
\text { (Node 7501 filtered @180 Hz) } \\
\end{array}$ & 130 g's & $90 \mathrm{~g}$ 's & 120 g's & $90 \mathrm{~g}$ 's & $161 \mathrm{~g}$ 's & $126 \mathrm{~g}$ 's \\
\hline $\begin{array}{c}\text { Neck force } \\
\text { (Max JT2-5 filtered @180Hz) } \\
\end{array}$ & $6806 \mathrm{~N}$ & $3950 \mathrm{~N}$ & $3500 \mathrm{~N}$ & $3950 \mathrm{~N}$ & $3264 \mathrm{~N}$ & $3283 \mathrm{~N}$ \\
\hline $\begin{array}{c}\text { Neck moment flexion } \\
\text { (Max +JSR17 phi, theta, psi } \\
\text { filtered @180Hz) } \\
\end{array}$ & $310 \mathrm{Nm}^{* *}$ & $315 \mathrm{Nm}$ & $165 \mathrm{Nm}$ & $142 \mathrm{Nm}$ & $123 \mathrm{Nm}$ & $84 \mathrm{Nm}$ \\
\hline $\begin{array}{l}\text { Neck moment extension } \\
\text { (Max + JSR71 phi, theta, psi } \\
\text { filtered @ @180Hz) }\end{array}$ & $135 \mathrm{Nm}^{* *}$ & $180 \mathrm{Nm}$ & $130 \mathrm{Nm}$ & $125 \mathrm{Nm}$ & $132 \mathrm{Nm}$ & $96 \mathrm{Nm}$ \\
\hline
\end{tabular}

* All configurations utilize five point harness

${ }^{* *}$ Frontal impact criteria used for lateral direction

Table 2. Comparison Among Dummy Configurations Using Input Acceleration Profile that Produces High Brinkley Injury in Lateral (Sideways) Direction.

The second configuration is where the crewmember is constrained in the five-point harness and thin side padding is placed three inches away from the head and shoulders. The large excursions of the head, arms and legs that were seen for the configuration without side padding is eliminated with the thin pad configuration. The results in Table 2 show that the thin side pads help to reduce the neck moments, which was expected since the side pads prevent the head from bending over. However, the Head Injury Criteria (HIC), CSI and thorax and pelvis acceleration criteria all exceed their allowable limits and are considerably larger than for the configuration without any pads. These results were also expected since the crewmember head and chest impact into the relatively thin and stiff pads leading to short durations of very large accelerations.

The configuration with the thicker pads and one-inch gap between the pads and head and chest provides a higher level of injury protection than the previous two cases. For this configuration, only the chest severity index and the thorax maximum allowable acceleration are exceeded. In fact, the thicker pads provide so much reduction in head acceleration that the head injury criterion is reduced by an order of magnitude over the configuration with thin pads. For the thick pad configuration where the pads are in contact with the head and shoulders, the head injury criteria and the chest severity index are further reduced however; the pelvis acceleration is increased and is beyond the allowable limit. For the thick pads and constrained arm and leg configuration, all of the observed injury criteria are reduced compared to the other configurations except for the chest severity index and the thorax acceleration. Both of these criteria exceed their allowable limits.

In general, introduction of the side supports eliminates the large excursion of the head and the hyperextension of the neck. Neck hyperextensions are the main cause of severe injury for the case without lateral support, so it appears promising that lateral support will improve survivability for $\mathrm{Y}$-axis direction impact. However the body must still mitigate the crash, so while neck forces are reduced due to reduced head and neck extension, other body forces and moments may show increases. This is supported by the fact that padding had the effect of reducing the neck moment while increasing the chest severity index. The key design goal is to keep any individual 
force, moment or acceleration from exceeding the allowable injury level, which will require tradeoffs in the design. With further design improvements it may be possible to reduce the chest and thorax loadings to within allowable limits, however, for the designs that were considered, both the Brinkley model and the FE crash test dummy predicted high levels of injury.

\section{X-Axis (Chest In/Out) Acceleration Simulations and Results}

A comparison between the Brinkley model and the FE crash test dummy was made in the $\mathrm{X}$-axis direction using an applied acceleration profile obtained from a maximum drag abort situation. This situation occurs when an emergency occurs during ascent and the crew module must be separated from the launch vehicle using the Launch Abort System (LAS). When the LAS is activated, the crewmembers are pushed into the backs of their seats while the LAS rockets are fired. Once the LAS rocket's fuel is depleted they cease firing and aerodynamic drag reverses the acceleration on the crewmembers and they are pulled out of their seats in the opposite direction. The Hybrid III FE crash test dummy is probably most suited for loadings in the $+\mathrm{X}$-axis direction since this is the direction where the test dummy was originally designed for automotive frontal impacts.

The acceleration profile for the maximum drag abort is shown in Figure 10 and the corresponding FE crash test dummy response at intermediate time steps is shown in Figure 11. As expected, during LAS rocket firing the crewmember is pushed into his seat and there is minimal motion of the head, arms or legs. In the later stage when aerodynamic drag is dominant and the crew member is pulled out of his seat, both the head and arms and legs are extended leading to the potential for larger neck forces and head accelerations. (Note: this particular model contained overly stiff elbow joints which explains the limited extension of the arms) Additionally, flailing of the arms and legs in the close confines of Orion could be an issue since the arms and legs may impact surrounding structures or strike other crewmembers. To compensate for flailing, hand and foot restraints will be important. The large head movement could also be an issue and some type of head/helmet constraint may be required.

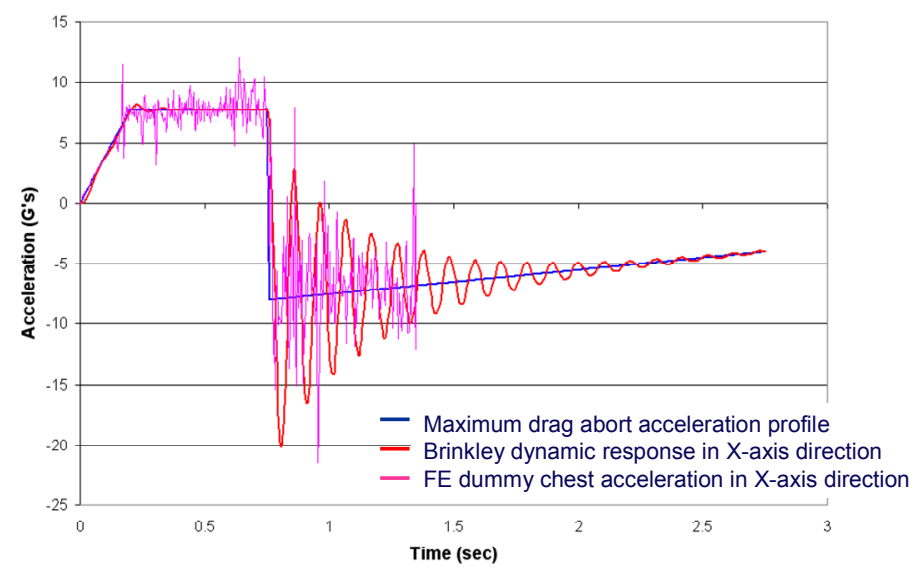

Figure 10. Acceleration Profile for Maximum Drag Abort, Brinkley Dynamic Response and FE Dummy Chest Acceleration. 


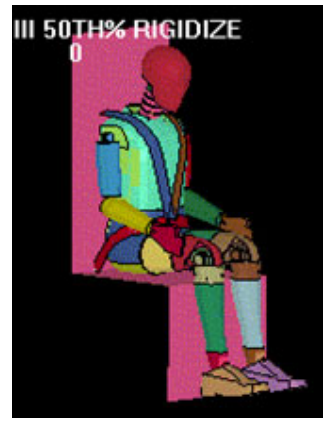

Initial Conditions

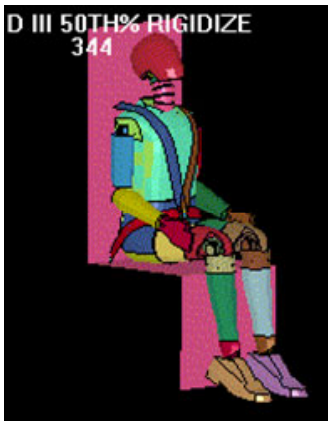

Large Abort System Rocket Firing

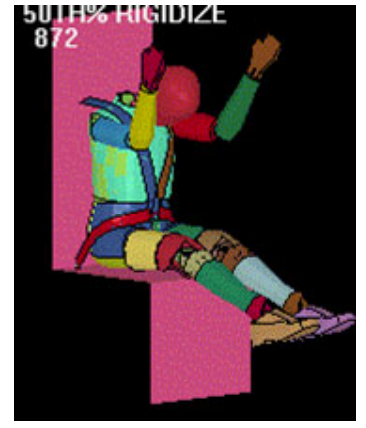

Aerodynamic Drag

Figure 11. Hybrid III Crash Test Dummy Response at Intermediate Time Steps for Maximum Drag Abort.

Figure 10 shows the maximum drag abort acceleration that was applied to the crewmember seat along with the resulting Brinkley dynamic response and the FE crash test dummy response in the $\mathrm{X}$-axis direction. Both the Brinkley and the dummy response follow, reasonably close, both the input acceleration shape and magnitude. The Brinkley response very closely tracks the input acceleration profile during the rocket-firing phase then oscillates about the input acceleration during the aerodynamic drag phase. The Brinkley model, as mentioned previously, employs an approximately $10 \mathrm{~Hz}$ oscillator, so it is expected that the response after the load reversal would oscillate and that the oscillations would be near $10 \mathrm{~Hz}$. The maximum Brinkley dynamic response is $20 \mathrm{G}$, which is within the Brinkley low risk of injury range for $\mathrm{X}$-axis (chest in/out) accelerations. The FE crash test dummy response is within the same general range as the Brinkley response except for numerous short duration acceleration spikes through the transient response. The dummy response has been filtered to eliminate response above $180 \mathrm{~Hz}$ and further reductions, or elimination, of at least some of these acceleration spikes could be eliminated by adding more damping to the dummy model and refinement of the model through the inclusion of a higher fidelity seat model and a small amount of seat padding. Even with these outlying acceleration peaks, the computed Chest Severity Index (CSI) is well below the allowable limit $(212<700)$ leading to a consistent result with the Brinkley prediction of a low probability of injury.

\section{Conclusions and Recommendations}

The FE crash test dummies used in conjunction with the Brinkley model provides a useful set of tools for predicting potential crew injuries during vehicle landings. In addition these tools enable the design and evaluation of alternate crew protection systems. Specifically:

- The Brinkley model reasonably ensures an acceptable environment for crew members in a five point harness; however, it has limited ability to provide insight into additional or modified crew restraint and protection systems. The Brinkley Model has been modified to included the general effect of sideways crew member support, however, the Brinkley Model does not distinguish between types of support or their differences in effectiveness in preventing injury. 
- FE analysis and crash test dummy tests are capable of providing valuable insight into alternate crew member injury protection systems and should be employed for this purpose. The FEA model and the physical crash test dummies can be used to assess the effects of variations of restraint and support in a comparative manner and if validated with human response data, can provide quantitative assessments of crew injuries.

- Additional evaluation of automobile industry safety standard injury criteria should be initiated to ascertain the applicability of these criteria to crew member protection.

- Practical methods for implementing additional crew protection appears realistic and should be further developed.

\section{References}

Bowman, B. M., Aircrew Ejection Injury Analysis and Trauma Assessment Criteria. Final report, University of Michigan, Ann Arbor, Transportation Research Institute, UMTRI-93-8, 1993.

Brinkley, J.W., Development of Aerospace Escape Systems, Air University Review, Vol. XIX (5):34-49, July-August 1968

Brinkley, J.W., and Shaffer, J.T., Dynamic Simulation Techniques for the Design of Escape Systems: Current Applications and Future Air Force Requirements, Aerospace Medical Research Laboratory Report AMRL TR 71-292, Wright Patterson Air Force Base, Ohio, December 1971.

Coltman, J.W. et al., Aircraft Crash Survival Design Guide-Volume II-Aircraft Design Crash Impact Conditions and Human Tolerance, USAAVSCOM TR 89-D228, December 1989.

Gramling, H., Hodgman, P., Hubbard, R. Development of the HANS head and neck support for Formula One. In: 1998 Motor sports engineering conference proceedings. SAE 983060. Warrendale, PA: Society of Automotive Engineers, 1998.

Melvin, J.W., Begemen, P.C., Faller, R.K. et al. Crash protection of stock car racing drivers - application of biomechanical analysis of Indy car crash research. Stapp Car Crash Journal 2006;50:415-28.

NASA Johnson Space Center, Constellation Program, 2006. Human-Systems Integration Requirements, CxP70024.

Schmitt, K.-U., Niederer, P., and Walz, F., Trauma Biomechanics: Introduction to Accidental Injury. Springer-Verlag, New York, New York, 2004.

Stech, E.L. and Payne, P.R., Dynamic Models of the Human Body, AAMRL-TR-66157, Aerospace Medical Research Laboratory, Wright-Patterson Air Force Base, Ohio, 1966.

von Gierke, H.E., Response of the Body to Mechanical Forces-An Overview, Lectures in Aerospace Medicine, $6^{\text {th }}$ Series, School of Aerospace Medicine, Brooks Air Force Base, Texas, pp.325-344, 1967. 